Original Research

\title{
Eplet-Based HLA Class II Matching for Transplantation: Design of a Repertoire of Interlocus Eplets Shared between HLA-DR, -DQ and -DP Alleles
}

\author{
Rene J. Duquesnoy ${ }^{*}$, Marilyn Marrari
}

Thomas E. Starzl Transplantation Institute University of Pittsburgh Medical Center, Pittsburgh, PA USA; E-Mails: Duquesnoyr@upmc.edu; mmarr@upmc.edu

* Correspondence: Rene. J. Duquesnoy; E-Mail: Duquesnoyr@upmc.edu

Academic Editor: Haval Shirwan

Special Issue: Antibody Mediated Rejection in Organ Transplantation

OBM Transplantation

2020, volume 4, issue 1

doi:10.21926/obm.transplant.2001099
Received: December 13, 2019

Accepted: February 06, 2020

Published: February 11, 2020

\begin{abstract}
Many studies have demonstrated that HLA-DR, HLA-DQ and HLA-DP matching at the eplet level reduces allograft rejection and improves transplant outcome. Such studies have examined the eplet effect for the individual class II loci, but until now little attention has been given to so-called interlocus class II eplets shared between HLA-DR, HLA-DQ and/or HLA-DP alleles. This report summarizes current information about antibody-verified interlocus class II eplets. It describes a structural modeling method to determine potentially immunogenic interlocus class II eplets and to identify non-immunogenic eplets because they are monomorphic at another class II locus. We propose that the inclusion of interlocus class II eplets will enhance the efficiency of eplet-based HLA-DR, -DQ, -DP matching in organ transplantation.
\end{abstract}

\section{Keywords}

HLA matching; eplet; HLA-DRDQDP; interlocus; HLAMatchmaker; HLA antibodies

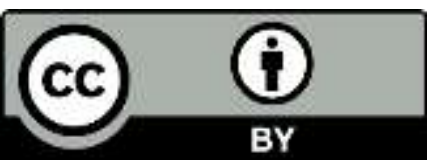

(C) 2020 by the author. This is an open access article distributed under the conditions of the Creative Commons by Attribution License, which permits unrestricted use, distribution, and reproduction in any medium or format, provided the original work is correctly cited. 


\section{Introduction}

There are now many reports that HLA-DR, HLA-DQ and HLA-DP matching at the eplet level is associated with reduced allograft rejection and better outcome of kidney, heart, lung, pancreas and liver transplants [1-21]. These studies have examined the eplet effect for the individual class II loci, but we must raise the question that certain class II eplets are shared between HLA-DR, HLADQ and/or HLA-DP alleles, the so-called interlocus class II eplets. This concept is analogous to sharing of antibody-verified class I eplets by combinations of HLA-A, -B and/or -C alleles such as 62GE shared between $A 2$ and $B 17,82 L R$ shared between $A 23, A 24, A 25, A 32$ and Bw4-carrying HLA-B alleles and, $163 \mathrm{LW}$ shared between various HLA-A, $-B$ and $-C$ alleles. The HLA-ABC eplet repertoire of the International HLA Epitope Registry (http://www.epregistry.com.br) has additional antibody-verified and non-verified interlocus class I eplets.

HLA-DR matching at the eplet level considers DRB1 together with DRB3, DRB4 or DRB5 and several antibody-verified DRB eplets are shared between different DRB loci. Examples include $4 Q$ (on DRB1*07, DRB1*09 and DRB4*01), 74R (on DRB1*03 and DRB3*01:01) and 96EV (on DRB1*01, DRB5*01:01 and DRB5*02:02). Except for the well-documented, antibody-verified eplet shared between DR11 and a group of DPB alleles (details are below), there is little information about interlocus eplet sharing between DR, DQ and DP. Four-digit allele-based HLA typing for all class II loci is now readily available in histocompatibility laboratories and this permits a determination of the full HLA-DR, -DQ, -DP eplet repertoire.

This report describes our method to characterize interlocus class II eplets. Using aligned amino acid sequences of $\alpha$ or $\beta$ chains we have identified a group of interlocus eplets that are defined by one or two polymorphic residues uniquely shared between alleles controlled by combinations of DR, DQ and DP loci. Consistent with our previously reported molecular modelling strategy [22], we defined eplet structures by identifying with the Cn3D program all residues within a 3.5 Ångstrom radius of the polymorphic residue(s).

Besides describing studies presenting preliminary evidence of a few antibody-verified interlocus class II eplets, this report describes a structural modelling method to identify other interlocus eplets on class II $\beta$ chains and $\alpha$ chains as potential candidates for experimental verification with informative antibodies.

We also report our findings that certain eplets are defined residues that are polymorphic at one class II locus but are monomorphic at another class II locus. Such eplets may be considered donorrecipient matches and their corresponding interlocus eplets may not be immunogenic.

The inclusion of interlocus class II eplets offers opportunities that will increase the accuracy of HLA-DR, -DQ, -DP matching at the eplet level.

\section{Methods and Results}

\subsection{Comparisons of Amino Acid Sequences of DR, DQ and DP Chains}

The protein BLAST (Basic Local Alignment Search Tool) program [23] is a commonly used tool to determine sequence and structural similarities between different proteins. This program can be accessed at www.blast.ncbi.nlm and upon entering amino acid sequences of two proteins it generates the following information: (1) Statistical significance of the residue alignment between two proteins, (2) Identification of the number and percentage of sequence positions with identical 
residues, (3) the number and percentage of sequence positions with identical residues together with non-identical residues with positive BLOSUM scores and (4) the number of gaps in the sequence alignment.

In bioinformatics, BLOSUM (BLOcks SUbstitution Matrix) is used to score alignments between evolutionary divergent protein sequences [24]. This has been done by calculating a log-odds score for each of the 210 possible substitution pairs of the 20 standard amino acids in 2000 aligned blocks of 500 groups of related proteins. For each amino acid substitution between two aligned sequences, BLOSUM has scores as positive (see Table S1), zero or negative. For instance, leucine has positive BLOSUM scores with structurally similar isoleucine $(+2)$, methionine $(+2)$ and valine $(+3)$, a 0 score with phenylalanine and negative scores with the remaining residues such as alanine $(-1)$ and asparagine (-4). The BLOSUM matrix is considered a useful guide to study the evolutional and chemical relationship between various proteins [25].

A BLAST analysis of HLA-DR, HLA-DQ and HLA-DP proteins has, as expected, a very high degree of sequence homology. As an example, Table S2 shows the results for class II $-\beta$ chains: DRB1*01:01 versus DQB1*02:01, DRB1*01:01 versus DPB1*01:01 and DQB1*02:01 versus DPB1*01:01. In all cases the structural homology was statistically highly significant because the $p$ values are less than $1 \times 10^{-90}$. The numbers of identical residue combinations in the amino acid 1190 sequence ranged between 127 to 138 (67\% to 73\%). About $80 \%$ of the residues had a positive alignment; they included 22, 24 and 14 non-identical residue pairs with positive BLOSUM scores (indicated by a "+" sign). BLAST analyses with other DRB, DQB and DPB allelic combinations yielded similar results (data not shown). The HLA-DP sequences show gaps in sequence positions 24 and 25 when compared with HLA-DRB and HLA-DQB and there is also a gap in position 154. These gaps in DPB lack amino acid residues present in DRB and DQB.

Table S3 shows the BLAST analysis data for class II $\alpha$ chains namely, DRA1*01:01, DQA1*01:01 and DPA $1 * 01: 03$. The frequencies of identical residues ranged from $58 \%$ to $63 \%$, somewhat lower than for the $-\beta$ chains; $72 \%$ to $74 \%$ of the residues had a positive alignment. The sequences of DRA and DPA have gaps in positions 1,2 and 15 when compared with DQA sequences.

\subsection{Interlocus Eplet Assignments on 6 Chains}

Interlocus eplets were determined for the 1-190 sequences adjusted for residue identity positions on HLA-DRB, -DQB and -DPB chains. To account for the three gaps in the 1-190 sequence of HLA-DPB, adjustments were made whereby DPB positions 24-153 became 26-155 and DPB positions 154-190 became 157-193. Interlocus eplets were assigned by identifying in each sequence location one or more polymorphic residues shared between two or all three class II loci. Interlocus eplets were assigned with prefixes such as "rq", "rp", "qp" or "rqp", indicating the sharing between DRB, DQB and/or DPB alleles. This report addresses only class II alleles that are commonly used in antibody testing assays with single allele beads.

For all eplet-carrying alleles we have determined with the Cn3D molecular modelling program [26] which amino acid residues are located within a 3.5 Ångstrom radius of the residue(s) used to assess the dimension of an eplet [22]. For each interlocus eplet we have identified within that area, the most commonly shared residues with standard one-letter amino acid codes. For each allele, identical residues are displayed with dash symbols and different residues are shown with the one-letter amino acid codes. Residue differences with positive BLOSUM scores (Table S1) as 
indicators of a structural and evolutional relationship have been marked with an asterisk. For instance, the difference between alanine and serine has a +1 BLOSUM score and substitutions for $A$ are marked as $S^{*}$ and substitutions for $S$ are marked as $A^{*}$; other substitutions for these residues are marked with the usual single letter codes without an asterisk.

Interlocus eplets on class II $\alpha$ chains were also determined for DRA, DQA and DPA. They were based on the 1-190 sequence of DQA alleles and sequences of DRA and DPA were adjusted to DQA after identifying a gap in position 15 . Since DRA is largely monomorphic, interlocus eplets can only be between DQA and DPA alleles; they have the "qp" prefix.

\subsection{Antibody-Verified Interlocus rp58E and rp58EE Eplets}

The best documentation of an antibody-verified class II interlocus eplet is shared between DR11 and a group of DPB alleles. Table 1 describes two variations. Human and mouse monoclonal antibodies are specific for rp58E shared between DRB1*11:01/03/04 and DPB1*02, *03, *04:02, $* 06, * 09, * 10, * 14, * 16, * 17, * 18, * 20$ and $* 28$ [27-34]. All rp58E-carrying alleles have the same $54 \mathrm{G}, 55 \mathrm{R}, 56 \mathrm{P}, 57 \mathrm{D}, 60 \mathrm{Y}, 61 \mathrm{~W}$ and $62 \mathrm{~N}$ residues within a 3.5 Ångstrom radius and the reactivity of a $\mathrm{rp58E}$-specific antibody is unaffected by the presence of $59 \mathrm{D}^{*}$ which has a Blosum score of +2 when compared to 59E. Table 1 shows also a second antibody reactivity pattern defined by rp58EE which is shared between DRB1*11:01/03/04 and DPB1*02,*04:02,*10,*16, *18 and *28 [33, 35, 36]; this eplet is defined by residues $58 \mathrm{E}$ and $59 \mathrm{E}$ and it is apparent that for this antibody, a substitution by 59D* has a negative effect.

Table 1 Reactivity patterns of antibody-verified rp58E and rp58EE eplets and amino acid residues within a 3.5 Ångstrom radius.

\begin{tabular}{|c|c|c|c|c|c|c|c|c|c|c|c|}
\hline & $\begin{array}{l}54 \\
\text { G }\end{array}$ & $\begin{array}{c}55 \\
\mathbf{R}\end{array}$ & $\begin{array}{c}56 \\
P\end{array}$ & $\begin{array}{l}57 \\
\text { D }\end{array}$ & $\begin{array}{c}58 \\
\text { Residue }\end{array}$ & $\begin{array}{l}59 \\
E\end{array}$ & $\begin{array}{l}60 \\
Y\end{array}$ & $\begin{array}{l}61 \\
W\end{array}$ & $\begin{array}{l}62 \\
N\end{array}$ & $\begin{array}{c}\text { Antibod } \\
\text { Eplet }\end{array}$ & $\begin{array}{c}\text {-verified } \\
\text { Eplet }\end{array}$ \\
\hline DRB1*11:01 & - & - & - & - & E & - & - & - & - & rp58E & rp58EE \\
\hline DRB1*11:02 & - & - & - & - & $E$ & - & - & - & - & rp58E & rp58EE \\
\hline DRB1*11:04 & - & - & - & - & $\mathbf{E}$ & - & - & - & - & rp58E & rp58EE \\
\hline DPB1*02:01 & - & - & - & - & $\mathbf{E}$ & - & - & - & - & rp58E & rp58EE \\
\hline DPB1*04:02 & - & - & - & - & $E$ & - & - & - & - & rp58E & rp58EE \\
\hline DPB1*08:01 & - & - & - & - & $E$ & - & - & - & - & rp58E & rp58EE \\
\hline DPB1*10:01 & - & - & - & - & $\mathbf{E}$ & - & - & - & - & rp58E & rp58EE \\
\hline DPB1*16:01 & - & - & - & - & $E$ & - & - & - & - & rp58E & rp58EE \\
\hline DPB1*18:01 & - & - & - & - & $\mathbf{E}$ & - & - & - & - & rp58E & rp58EE \\
\hline DPB1*28:01 & - & - & - & - & $\mathbf{E}$ & - & - & - & - & rp58E & rp58EE \\
\hline DPB1*03:01 & - & - & - & - & $E$ & $D^{*}$ & - & - & - & rp58E & \\
\hline DPB1*06:01 & - & - & - & - & $\mathbf{E}$ & $D^{*}$ & - & - & - & rp58E & \\
\hline DPB1*09:01 & - & - & - & - & $E$ & $D^{*}$ & - & - & - & rp58E & \\
\hline DPB1*14:01 & - & - & - & - & $E$ & $\mathbf{D}^{*}$ & - & - & - & rp58E & \\
\hline DPB1*17:01 & - & - & - & - & $E$ & $D^{*}$ & - & - & - & rp58E & \\
\hline DPB1*20:01 & - & - & - & - & $\mathbf{E}$ & $\mathbf{D}^{*}$ & - & - & - & rp58E & \\
\hline
\end{tabular}




\subsection{Predictions of Other Interlocus Class II Eplets in the 54-62 Sequence}

This sequence has four additional interlocus eplets with a high degree of amino acid residue similarity. None have been verified experimentally with informative antibodies, but they might be suitable candidates. These eplets are defined by polymorphic residues in position 57 and the interlocus eplets are called rq57S, rqp57A, rq57V and rqp57D (Table 2). DQB1*05:02 shares rq57S with DRB1*04:05, *08:01 and *13:03 and the residues in the 54-62 sequence are identical. The rqp57A eplet is shared between DRB1*14:01, DQB1*02 and *03:02 and DPB1*01, *04:01, *11, $* 13, * 15$ and $* 23$ have considerable $54-62$ sequence similarity but it is possible that the difference in position 55 , i.e. $55 \mathrm{R}$ versus $55 \mathrm{~L}$ and/or $55 \mathrm{P}$ may affect cross-reactivity with antibody. The rq57V eplet shared between DRB1*07, *09, *12, DRB3*01:01, *03:01 and DQB1*05:01, *06:04/09 have identical residues in positions $54,55,56,58,59,61$ and 62 ; the only difference is at position 60 whereby the DRB alleles have $60 \mathrm{~S}$ and the DQB alleles have 60Y.

Table 2 Allele sharing for four interlocus eplets in sequence position 57 and amino acid residues within a 3.5 Ångstrom radius.

\begin{tabular}{|c|c|c|c|c|c|c|c|c|c|c|c|c|c|c|c|c|c|c|c|}
\hline & 54 & 55 & $\begin{array}{lll}56 & 57 & 5\end{array}$ & 5 & 960 & 61 & & & & & 45 & & 57 & & 59 & o & 61 & 62 & \\
\hline & G & $\mathbf{R}$ & $P$ sid $A$ & E & $Y$ & W & $\mathbf{N}$ & Eplet & & ( & $\mathrm{R}$ & & Residue & $A$ & $\mathrm{E}$ & $\mathbf{Y}$ & w & $\mathbf{N}$ & Eplet \\
\hline DRB1*04:05 & - & - & $-57 S$ & . & - & - & - & rq57S & DRB1*01 & & - & . & 57D & - & - & - & - & -1 & rqp57D \\
\hline DRB1 ${ }^{*} 08: 01$ & - & - & $-57 S$ & . & - & - & - & rq57s & DRB1*03 & & - & . & 57D & - & - & - & - & - & rqp57D \\
\hline DRB1 ${ }^{*} 13: 03$ & - & - & $-57 S$ & . & - & - & - & rq57s & DRB1 ${ }^{*} 04: 01$ & & - & . & 57D & - & - & - & - & - & rqp57D \\
\hline DQB1 ${ }^{*} 05: 02$ & - & & $-57 S$ & . & - & - & - & rq57S & DRB1 ${ }^{*} 04: 02$ & t. & - & . & 57D & - & - & - & - & - & rqp57D \\
\hline & & & & & & & & & DRB1 ${ }^{*} 04: 03$ & & - & . & 57D & - & - & - & - & - & rqp57D \\
\hline & & & & & & & & & DRB1*04:04 & & - & . & 57D & - & - & - & - & - & rqp57D \\
\hline DRB1*14:01 & - & - & - 57A. & & $\mathrm{H}^{*}$ & - & - & rqp57A & DRB1 ${ }^{*} 08: 02$ & s. & - & . & 57D & - & - & - & - & - & rqp57D \\
\hline DQB1 ${ }^{*} 02: 01$ & - & L & $-57 A$ & & - & - & - & rqp57A & DRB1 ${ }^{*} 09: 02$ & . & - & . & 57D & - & - & - & - & - & rqp57D \\
\hline DQB1 ${ }^{*} 02: 02$ & - & L & $-57 A$ & & - & - & - & rqp57A & DRB1*10:01 & 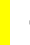 & - & . & 57D & - & - & - & - & - & rqp57D \\
\hline DQB1 ${ }^{*} 03: 02$ & - & $\mathbf{P}$ & $-57 A$ & . & - & - & - & rqp57A & DRB1*11:01 & & - & . & 57D & $\mathrm{E}$ & - & - & - & - & rqp57D \\
\hline DPB1 ${ }^{*} 01: 01$ & - & - & $-57 A$ & . & - & - & - & rqp57A & DRB1*13:01 & s. & - & . & 57D & - & - & - & - & - & rqp57D \\
\hline DPB $1{ }^{\star} 04: 01$ & - & - & $-57 A$ & & - & - & - & rqp57A & DRB1*13:02 & 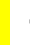 & - & . & 57D & - & - & - & - & - & rqp57D \\
\hline DPB1*11:01 & - & - & $-57 A$ & & - & - & - & rqp57A & DRB1*14:02 & . & - & . & 57D & - & - & - & - & - & rqp57D \\
\hline DPB1*13:01 & - & - & $-57 A$ & . & - & - & - & rqp57A & DRB1*14:54 & & - & . & 57D & - & - & $\mathrm{H}^{*}$ & - & - & rqp57D \\
\hline DPB1*15:01 & - & - & $-57 A$ & & - & - & - & rqp57A & DRB1*15 & 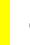 & - & . & 57D & - & - & - & - & - & rqp57D \\
\hline DPB1*23:01 & - & & $-57 A$ & & - & - & - & rqp57A & DRB1*16 & 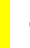 & - & . & 57D & - & - & - & - & - & rqp57D \\
\hline & & & & & & & & & DRB3*02:02 & & - & . & 57D & - & - & - & - & - & rqp57D \\
\hline DRB1 ${ }^{*} 07: 01$ & - & - & $-57 \mathrm{~V}$ & & $\mathbf{s}$ & - & - & rq57V & DRB4* & se & - & . & 57D & - & - & - & - & - & rqp57D \\
\hline DRB1 ${ }^{*} 09: 01$ & - & - & $-57 \mathrm{~V}$ & & $\mathbf{s}$ & - & - & rq57V & DRB5* & 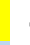 & - & . & 57D & - & - & - & - & - & rqp57D \\
\hline DRB1 ${ }^{*} 12: 01$ & - & - & $-57 \mathrm{~V}$ & & $\mathbf{S}$ & - & - & rq57V & DQB1*03:01/03 & i & $\mathbf{P}$ & . & 57D & - & - & - & - & - & rqp57D \\
\hline DRB1*12:02 & - & - & $-57 \mathrm{~V}$ & & $\mathbf{s}$ & - & - & rq57V & DQB1*04 & & - & $\mathrm{t}$ & 57D & - & - & - & - & - & rqp57D \\
\hline DRB3*01:01 & - & - & $-57 \mathrm{~V}$ & & $\mathbf{s}$ & - & - & rq57V & DQB1*06:01/02/03 & & - & . & 57D & - & - & - & - & - & rqp57D \\
\hline DRB3*03:01 & - & - & $-57 \mathrm{~V}$ & & $\mathbf{s}$ & - & - & rq57V & DPB1*02:01 & t & - & . & 57D & E & - & - & - & -1 & rqp57D \\
\hline DQB1 ${ }^{*} 05: 01$ & - & & $-57 \mathrm{~V}$ & & - & - & - & rq57V & DPB1*03:01 & & - & . & 57D & E & $D^{*}$ & - & - & -1 & rqp57D \\
\hline DQB1 ${ }^{*} 06: 04$ & - & - & $-57 \mathrm{~V}$ & & - & - & - & rq57V & & & - & . & 57D & $\mathbf{E}$ & - & - & - & - & rqp57D \\
\hline DQB1*06:05 & - & - & $-57 \mathrm{~V}$ & & - & - & - & rq57V & DPB1*06:01 & & - & . & 57D & E & $D^{*}$ & - & - & - & rqp57D \\
\hline DQB1 ${ }^{*} 06: 09$ & - & - & $-57 \mathrm{~V}$ & & - & - & - & rq57V & DPB1 ${ }^{*} 08: 01$ & & - & . & 57D & E & - & - & - & -1 & rqp57D \\
\hline & & & & & & & & & DPB1 ${ }^{*} 09: 01$ & & - & & 57D & E & $D^{*}$ & - & - & -1 & rqp57D \\
\hline & & & & & & & & & DPB1 & & & & 57D & $\mathbf{E}$ & - & & - & - & rqp57D \\
\hline & & & & & & & & & & & & & 57D & $\mathrm{E}$ & $D^{*}$ & & - & - & rqp57D \\
\hline & & & & & & & & & DPB1*17:01 & . & - & . & 57D & E & $D^{*}$ & - & - & -1 & rqp57D \\
\hline & & & & & & & & & DPB1*18:01 & & - & . & 57D & E & - & - & - & -1 & rqp57D \\
\hline & & & & & & & & & & & & & 57D & E & $\mathbf{D}^{*}$ & & - & - & rqp57D \\
\hline & & & & & & & & & DPB1*28:01 & & - & & 57D & E & - & - & - & -1 & rqp57D \\
\hline
\end{tabular}


Many DRB alleles share rqp57D, and 7 of 8 additional residues with DQB1*03:01 and *03:03 which have $55 \mathrm{P}$ instead of $55 \mathrm{R}$, and $\mathrm{DQB} 1 * 04$ which has $56 \mathrm{~L}$ instead of $56 \mathrm{P}$. It should be noted that the rqp57-carrying DQB1*06 alleles have identical residues as the rqp57D-carrying DRB alleles. All rqp57D-carrying DPB alleles have 58E instead of 58A and about half of them have 59D* instead of 59E. It is possible that the reactivity of rqp57D might be affected by residue differences at DPB and DQB1*03:01/03 and DQB1*04 and that the interlocus eplet is shared only between DRB alleles and DQB1*06:01/02/03 that have identical residues within a 3.5 Ångstrom radius.

Given their interlocus structural similarity, it seems that rq57S, rqp57A, rq57V and rqp57D might be considered as potential candidates for experimental verification with informative antibodies.

\subsection{Have Other Interlocus Class II Eplets Been Antibody-Verified?}

Current information about antibody-verified interlocus class II eplets is quite limited but a few studies have yielded promising data which must nevertheless be considered preliminary. The experimental evidence for three antibody-verified interlocus class II eplets has been summarized as follows:

\subsubsection{Antibody-Verified rq70RK/R on DQ2, DR9, DR10, DRB1*14:01 and Possibly DR53.}

Drover et al [37] described a mouse monoclonal antibody NFLD.M71 that reacted in binding assays using homozygous cell lines with a polymorphic determinant shared between DQB1*02:01 chains and DRB1*14:01, DR9 and DR10. From an amino acid sequence analysis, it appears that NFLD.M71 recognizes a structural determinant determined by residues $70-73$, RKRA on DQBI*02:01 chains and RRRA on DRBI*09:01/02, *10:01, and *14:01 chains. Our analysis suggests the interlocus rq70RK/R eplet defined by residues $70 R+71 K^{*}$ on $D Q B$ or $70 R+71 R^{*}$ on DRB (Table 3 ). Six of seven positions within a 3.5 Ångstrom radius have identical residues. No information was provided about the reactivity of DRB4 alleles which also carry rq70R+71R*.

Table 3 Antibody-verified interlocus eplet rq70RK/R and amino acid residues within 3.5 Ångstroms.

\begin{tabular}{|c|c|c|c|c|c|c|c|c|c|}
\hline & NFLD.M71 & 6869 & 7071 & 72 & 73 & 74 & 75 & 76 & \\
\hline Allele & Antibody & L $E$ & Residues & $\mathbf{R}$ & A & E & v & D & Eplet \\
\hline DQB1*02:01 & Reactive & - & 70R71K* & - & - & A & - & - & $\mathrm{rq70RK/R}$ \\
\hline$D Q B 11^{\star} 02: 02$ & Not tested? & - & 70R71K* & - & - & A & - & - & rq70RK/R \\
\hline DRB1*09:01 & Reactive & - & 70R71R* & - & - & - & - & - & rq70RK/R \\
\hline DRB1*09:02 & Reactive & - & 70R71R* & - & - & - & - & - & rq70RK/R \\
\hline DRB1*10:01 & Reactive & - & 70R71R* & - & - & A & - & - & rq70RK/R \\
\hline DRB1*14:01 & Reactive & - & 70R71R* & - & - & - & - & - & rq70RK/R \\
\hline DRB4*01:01 & Not tested? & - & 70R71R* & - & - & - & - & - & rq70RK/R \\
\hline DRB4*01:03 & Not tested? & - & 70R71R* & - & - & - & - & - & rq70RK/R \\
\hline
\end{tabular}




\subsubsection{Antibody-Verified rp67IE on DRB1*01:03 and Several DP Alleles}

Callender et al [38] reported allosera with strong cross-reactivity between DP and DRB1*01:03 but not with other DRB1*01 alleles. Sequence alignments suggested a new cross-reactivity between DRB1*01:03 and DPB1*02,*09,*10,*13,*16 and *17. Two additional sera confirmed this cross-reactivity, although no data were presented about antibody reactivity patterns with the HLA panel. This epitope was originally described as $651+67 E$ on DP and $671+69$ E on DR but according to the new sequence alignment described above the interlocus eplet can be called rp67IE as defined by residues $67 \mathrm{I}$ and $71 \mathrm{E}$.

Eight residues within a 3.5 Ångstrom radius are identical between DRB1*01:03 and the rp67IEcarrying DPB1 alleles and the difference between 70D and 70E was acceptable for antibody reactivity (Table 4). The original report [38] indicated that the rp67IE-carrying DRB1*13:01 was weakly reactive; they have a nearby distinct $28 \mathrm{D}$ residue below the molecular surface but close enough to $67 \mathrm{I}$ and $71 \mathrm{E}$ to influence reactivity with this antibody. Table 4 also explains why DRB1*01:01 and DRB1*01:02 were non-reactive; these alleles carry a different eplet, 67LR.

Table 4 Antibody-verified interlocus eplet rp67IE and amino acid residues within 3.5 Ångstroms.

\begin{tabular}{|c|c|c|c|c|c|c|c|c|c|c|c|c|}
\hline & $\begin{array}{l}\text { Antibody } \\
\text { Reactive }\end{array}$ & $\begin{array}{l}646 \\
Q\end{array}$ & $\begin{array}{l}65 \\
K\end{array}$ & $\begin{array}{l}66 \\
D\end{array}$ & $\begin{array}{c}6771 \\
\text { Residues }\end{array}$ & $\begin{array}{c}68 \\
L\end{array}$ & $\begin{array}{l}69 \\
E\end{array}$ & $\begin{array}{l}70 \\
E\end{array}$ & $\begin{array}{c}72 \\
\mathbf{R}\end{array}$ & $\begin{array}{l}73 \\
\text { A }\end{array}$ & $\begin{array}{c}28 \\
E\end{array}$ & Eplet \\
\hline DPB1*02:01 & Positive & - & - & - & 67IE & - & - & - & - & - & - & rp67IE \\
\hline DPB1*09:01 & Positive & - & - & - & 67IE & - & - & - & - & - & - & rp67IE \\
\hline $\mathrm{DPB}^{*}{ }^{*} 10: 01$ & Positive & - & - & - & 67IE & - & - & - & - & - & - & rp67IE \\
\hline DPB1*13:01 & Positive & - & - & - & 67IE & - & - & - & - & - & - & rp67IE \\
\hline DPB1*16:01 & Positive & - & - & - & 67IE & - & - & - & - & - & - & rp67IE \\
\hline DPB1*17:01 & Positive & - & - & - & 67IE & - & - & - & - & - & - & rp67IE \\
\hline DRB1*01:03 & Positive & - & - & - & 67IE & - & - & $D^{*}$ & - & - & - & rp67IE \\
\hline DRB1*13:01 & Weak & - & - & - & 67IE & - & - & $D^{*}$ & - & - & $\mathbf{D}^{*}$ & rp67IE \\
\hline DRB1*01:01 & Negative & - & - & - & 67LR & - & - & $\mathbf{Q}^{*}$ & - & - & - & \\
\hline $\begin{array}{l}\text { DRB1*01:02 } \\
\text { Other DR orDP }\end{array}$ & $\begin{array}{l}\text { Negative } \\
\text { Negative }\end{array}$ & - & - & - & 67LR & - & - & $\mathbf{Q}^{*}$ & - & - & - & \\
\hline
\end{tabular}

\subsubsection{Antibody-Verified rq75VT on Several DRB Alleles and DQ4.}

The IgM human monoclonal antibody JOK1H7 originating in Arend Mulder's Laboratory at Leiden University Medical Center reacts with 75VT-carrying DRB alleles and additionally, with DQ4 heterodimers, which also have 75VT. All other DQ dimers were negative. This antibody originated from a woman who types as DRB1*03:01, *13:01, DRB3*01:01, DQB1*02:01, *06:03 and she was HLA sensitized during pregnancy. Although the HLA type of the child is unknown, her husband types as DRB1*04:02, *11:01, DRB3*02:02, DRB4*01:03, DQB1*03:02. This monoclonal antibody reacted with husband's DRB4*01:03 (MFI=7349) and DRB1*11:01 (MFI=3664); both have rq75VT.

Table 5 displays the MFI data with the entire DR and DQ panel. None of the DP heterodimers were reactive (data not shown). Positive reactions with DRB alleles were limited to 75VT-carrying DRB alleles except six alleles that have the combination of nearby residues $67 \mathrm{I}$ and $70 \mathrm{D}$. This suggests that the epitope defined by 75VT includes residues in sequence positions 67 and 70 ; the 
presence of $671+70 \mathrm{D}$ abolishes the reactivity with this antibody. We noted significantly lower MFI values (3551 versus 11792, $\mathrm{p}<0.00001$ ) for the seven 75VT-carrying DRB alleles that have 70D together with $67 \mathrm{~F}$ or $67 \mathrm{~L}$. This suggests that the presence of $70 \mathrm{D}$ also has some inhibitory effect. In contrast, the four DRB alleles which have $671+70 Q$ gave similarly high MFI values and the remaining 75VT-carrying DRB alleles also reacted well regardless of residue differences in nearby positions 67 ( $L$ and $F$ ), 70 ( $R$ and $Q^{*}$ ), $71\left(R, K^{*}\right.$ and $A$ ) and 74 ( $A$ and $E$ ). Other nearby positions 24, $72,73,76,79$ and 80 shared the same residue.

Table 5 Antibody reactivity of rq75VT presented by the immunizing DRB and crossreactive with DQ4.

\begin{tabular}{|c|c|c|c|c|c|c|c|c|c|c|c|c|c|c|}
\hline & & & 24 & 67 & 70 & 71 & 72 & 73 & 74 & 7577 & 76 & 78 & 79 & 80 \\
\hline & MFI & Eplet & V & $\mathbf{L}$ & $\mathbf{R}$ & $\mathbf{R}$ & $\mathbf{R}$ & A & A & Residues & D & $\mathbf{Y}$ & C & $\mathbf{R}$ \\
\hline DRB1*01:01 & 13223 & rq75VT & - & - & $\mathbf{Q}^{*}$ & - & - & - & - & 75V77T & - & - & - & - \\
\hline DRB1*01:02 & 12155 & rq75VT & - & - & $\mathbf{Q}^{*}$ & - & - & - & - & 75V77T & - & - & - & - \\
\hline DRB1*04:01 & 12568 & rq75VT & - & - & $\mathbf{Q}^{*}$ & $\mathbf{K}^{*}$ & - & - & - & 75V77T & - & - & - & - \\
\hline DRB1*04:03 & 11100 & rq75VT & - & - & $Q^{*}$ & - & - & - & E & 75V77T & - & - & - & - \\
\hline DRB1*04:04 & 13067 & rq75VT & - & - & $Q^{*}$ & - & - & - & - & 75V77T & - & - & - & - \\
\hline DRB1*04:05 & 12850 & rq75VT & - & - & $Q^{*}$ & - & - & - & - & 75V77T & - & - & - & - \\
\hline DRB1*09:01 & 12646 & rq75VT & - & $\mathbf{F}$ & - & - & - & - & E & 75V77T & - & - & - & - \\
\hline DRB1*09:02 & 13784 & rq75VT & - & $F$ & - & - & - & - & E & 75V77T & - & - & - & - \\
\hline DRB1*10:01 & 13610 & rq75VT & - & - & - & - & - & - & - & 75V77T & - & - & - & - \\
\hline DRB1*14:01 & 13202 & rq75VT & - & - & - & - & - & - & E & 75V77T & - & - & - & - \\
\hline DRB1*15:01 & 11790 & rq75VT & - & $I^{*}$ & $\mathbf{Q}^{*}$ & A & - & - & - & 75V77T & - & - & - & - \\
\hline DRB1*15:02 & 11544 & rq75VT & - & $I^{*}$ & $\mathbf{Q}^{*}$ & A & - & - & - & $75 \mathrm{~V} 77 \mathrm{~T}$ & - & - & - & - \\
\hline DRB1*15:03 & 12466 & rq75VT & - & $I^{\star}$ & $Q^{*}$ & A & - & - & - & 75V77T & - & - & - & - \\
\hline DRB4*01:01 & 6010 & rq75VT & - & - & - & - & - & - & E & 75V77T & - & - & - & - \\
\hline DRB4*01:03 & 7349 & rq75VT & - & - & - & - & - & - & E & 75V77T & - & - & - & - \\
\hline DRB5*02:02 & 11303 & rq75VT & - & $I^{*}$ & $\mathbf{Q}^{*}$ & A & - & - & - & 75V77T & - & - & - & - \\
\hline DRB1*16:01 & 5865 & rq75VT & - & $\mathbf{F}$ & D & - & - & - & - & 75V77T & - & - & - & - \\
\hline DRB1*12:02 & 5697 & rq75VT & - & $\mathbf{F}$ & D & - & - & - & - & 75V77T & - & - & - & - \\
\hline DRB1*16:02 & 4436 & rq75VT & - & - & D & - & - & - & - & 75V77T & - & - & - & - \\
\hline DRB1*11:01 & 3664 & rq75VT & - & $\mathbf{F}$ & D & - & - & - & - & 75V77T & - & - & - & - \\
\hline DRB1*11:04 & 2871 & rq75VT & - & $\mathbf{F}$ & D & - & - & - & - & 75V77T & - & - & - & - \\
\hline DRB1*08:01 & 1462 & rq75VT & - & $F$ & D & - & - & - & $\mathbf{L}$ & 75V77T & - & - & - & - \\
\hline DRB5*01:01 & 862 & rq75VT & - & $\mathbf{F}$ & D & - & - & - & - & 75V77T & - & - & - & - \\
\hline DRB1*13:03 & 402 & rq75VT & - & $I^{*}$ & D & $\mathbf{K}^{*}$ & - & - & - & 75V77T & - & - & - & - \\
\hline DRB1*12:01 & 65 & rq75VT & - & $I^{*}$ & D & - & - & - & - & 75V77T & - & - & - & - \\
\hline DRB1*01:03 & 19 & rq75VT & - & $I^{*}$ & D & $E$ & - & - & - & 75V77T & - & - & - & - \\
\hline DRB1*04:02 & 9 & rq75VT & - & $I^{*}$ & D & $E$ & - & - & - & 75V77T & - & - & - & - \\
\hline DRB1*13:01 & 7 & rq75VT & - & $I^{*}$ & D & $E$ & - & - & - & 75V77T & - & - & - & - \\
\hline DRB1*07:01 & 3 & rq75VT & - & $I^{*}$ & D & - & - & $\mathbf{G}$ & $\mathbf{Q}$ & 75V77T & - & - & - & - \\
\hline DQB1*04:01 & 5471 & rq75VT & - & $\mathbf{l}^{*}$ & $\mathbf{E}$ & D & - & - & $\mathbf{S}^{*}$ & 75V77T & - & v & - & - \\
\hline DQB1*04:02 & 3995 & rq75VT & - & $I^{*}$ & $E$ & D & - & - & $\mathbf{S}^{*}$ & 75V77T & - & v & - & - \\
\hline $\begin{array}{l}\text { non-75VT DRB } \\
\text { non-75VT DQB }\end{array}$ & $\begin{array}{c}6 \pm 2 \\
73 \pm 194\end{array}$ & & & & & & & & & & & & & \\
\hline
\end{tabular}

The panel has four heterodimers with $\mathrm{DQB1}$ *04:01 or DQB1*04:02; all were distinctly reactive with average MFI values of 5471 and 3995, respectively. DQB1*04 chains carry 75V77T together with $67 \mathrm{I}+70 \mathrm{E}$. In comparison with the rq75VT-carrying DRB alleles they have identical residues in 
positions $24,72,73,76,79$ and 80 . On the other hand, residues 70E, 71D, 745* and $78 \mathrm{~V}$ are distinct for DQB1*04; we cannot determine which residue(s) contribute to the lower MFI values with DQB1*04 as compared to the high MFI with many DRB alleles.

In conclusion, although this human monoclonal antibody must be considered specific for one epitope, its reactivity pattern with the allele panel was rather complex. The data suggest recognition of a rq75VT-related epitope shared between DRB and DQB.

\subsection{Predictions of Other Interlocus Class II Eplets on B Chains}

This report summarizes the experimental evidence for five antibody-verified interlocus class II eplets; their sequence positions within a 3.5 Ångstrom radius show high degrees of identical residue sharing (see Table 1, Table 3, Table 4 and Table 5). As described above (Table 2), the 54-62 sequence has four interlocus eplets that might be considered as suitable candidates for antibody verification. A search of the entire 1-190 sequence has identified a total of sixteen interlocus class II eplets with comparably similar degrees of residue sharing between eplet-carrying alleles. Table 6 lists these eplets and Table S4 describes the residues within a 3.5 Ångstrom radius. Certain interlocus eplets such as rq26Y, rq37YV and rqp57A are present on relatively small numbers of alleles whereas others are expressed by many alleles encoded by a given locus. Examples are rp37FV on most DPB alleles, rq57D on most DRB alleles and rqp67I on most DRB and DPB alleles. Such interlocus eplets have low probabilities of being mismatched and inducing specific antibodies.

We identified only one $\alpha$ chain interlocus eplet pq34Q shared between DPA1*02 and all DQA1 alleles except DQA ${ }^{*} 02$ (Table S4).

Table 6 DRB, DQB and DPB alleles with interlocus class II eplets that have not been antibody verified.

\begin{tabular}{|c|c|}
\hline \multicolumn{2}{|c|}{ Interlocus Eplet Luminex Alleles } \\
\hline rq26Y & DRB1 ${ }^{*} 03: 01,{ }^{*} 09: 01, \mathrm{DRB} 3{ }^{*} 01: 01, \mathrm{DQB} 1{ }^{*} 03: 01,{ }^{*} 06: 01$ \\
\hline \multirow{2}{*}{ rp37FV } & DRB1 ${ }^{*} 07: 01,{ }^{*} 14: 01,{ }^{*} 14: 54, D R B 3{ }^{*} 03: 01$ \\
\hline & DPB $1{ }^{*} 02,{ }^{*} 03,{ }^{\star} 04: 02,{ }^{*} 06,{ }^{\star} 08,{ }^{*} 09,{ }^{*} 10,{ }^{*} 14,{ }^{*} 16,{ }^{*} 17,{ }^{*} 18,{ }^{*} 19,{ }^{*} 20,{ }^{*} 23$ \\
\hline \multirow[t]{2}{*}{ rqp37YA } & DRB1*10,DRB3*02:02,DRB4, DQB1*03*04,*06:02/03/04/09, \\
\hline & DPB1*01, ${ }^{*} 11,{ }^{*} 13,{ }^{*} 15$ \\
\hline rq37Yv & DRB1 ${ }^{*} 04,{ }^{*} 08,{ }^{*} 11,{ }^{*} 13: 03$, DQB1 ${ }^{*} 05$ \\
\hline rqp57A & DRB1*14:01, DQB1 ${ }^{\star} 02,{ }^{*} 03: 02$, DPB $1{ }^{*} 01,{ }^{*} 04: 01,{ }^{*} 11,{ }^{*} 13,{ }^{*} 15,{ }^{*} 23$ \\
\hline \multirow[t]{3}{*}{ rqp57D } & DRB $1{ }^{*} 01,{ }^{*} 03,{ }^{*} 04: 01 / 02 / 03 / 04,{ }^{*} 08: 02,{ }^{*} 09: 02,{ }^{*} 10,{ }^{*} 11,{ }^{*} 13: 01 / 02,{ }^{*} 14: 02 / 54,{ }^{*} 15,{ }^{*} 16$ \\
\hline & DRB3*02:02,DRB4,DRB5, DQB1*03:01/03,*06:01/02/03 \\
\hline & DPB1 ${ }^{\star} 02,{ }^{*} 03,{ }^{*} 04: 02,{ }^{*} 06,{ }^{*} 08,{ }^{*} 09,{ }^{*} 10,{ }^{*} 14,{ }^{*} 17,{ }^{*} 18,{ }^{*} 28$ \\
\hline rq57S & DRB1 ${ }^{\star} 04: 05,{ }^{*} 08: 01,{ }^{*} 13: 03, \mathrm{DQB} 1{ }^{*} 05: 02$ \\
\hline rq57V & DRB1 ${ }^{*} 07: 01,{ }^{*} 09: 01,{ }^{*} 12, \mathrm{DRB} 3{ }^{*} 01: 01 / 03: 01, \mathrm{DQB} 1{ }^{*} 05: 01,{ }^{*} 06: 04 / 09$ \\
\hline qp67IE & DQB1 ${ }^{\star} 04$, DPB1 $1{ }^{*} 01,{ }^{*} 02,{ }^{*} 04,{ }^{*} 05,{ }^{*} 08,{ }^{*} 09,{ }^{*} 10,{ }^{*} 13,{ }^{*} 17,{ }^{*} 18,{ }^{*} 19,{ }^{*} 23$ \\
\hline rqp67IK & DRB1*13:03, DQB1*02, DPB1*01,*04, *05, *18, *23 \\
\hline rp67LK & DRB1 ${ }^{*} 03,{ }^{*} 04: 01$, DRB3, DPB $1{ }^{*} 03,{ }^{*} 14,{ }^{*} 28$ \\
\hline rp67LR & DRB1*01:01/02, *04:03/05, *10, *14, DRB4*01:01, DPB1*11 \\
\hline rq74AV & DRB1*01, ${ }^{\star} 04,{ }^{*} 10,{ }^{*} 11,{ }^{*} 12,{ }^{*} 13,{ }^{*} 14: 02,{ }^{*} 15,{ }^{*} 16$, DRB5, DQB1 ${ }^{*} 02$ \\
\hline qp77RV & DQB $1{ }^{*} 02,{ }^{*} 05$, DPB $1{ }^{*} 01,{ }^{*} 03,{ }^{*} 08,{ }^{*} 09,{ }^{*} 10,{ }^{*} 14$ \\
\hline rq77TV & DRB1*07:01, ${ }^{*} 09$, DQB1 ${ }^{*} 03,{ }^{*} 04,{ }^{*} 06$ \\
\hline rq140TV & DRB1 ${ }^{*} 03,{ }^{*} 04: 01,{ }^{*} 04: 02,{ }^{*} 04,{ }^{*} 08,{ }^{*} 10,{ }^{*} 11,{ }^{*} 12,{ }^{*} 13,{ }^{*} 14, \mathrm{DRB} 3{ }^{*} 03: 01, \mathrm{DQB} 1{ }^{*} 03,{ }^{*} 04$ \\
\hline
\end{tabular}




\subsection{Class II Eplets Defined by Polymorphic Residues that Are Monomorphic at Other Loci}

We have also searched the amino acid sequences of class II $\beta$ and $\alpha$ chains for eplets that are polymorphic for one locus but monomorphic for another locus. Such eplets might be considered non-immunogenic. This analysis focused on eplets defined by a shared polymorphic residue which is surrounded by at least one identical or structurally very similar residue on each side. Structural comparisons have considered the residues within a 3.5 Ångstrom radius to assess the degree of overall similarity between eplet-carrying alleles. Table S5 shows three DRB eplets (28E, 104S and $133 \mathrm{~L}$ ) and four DQB eplets (30YI, 45GE, 116V and 140A) that are monomorphic at another locus.

Table S6 shows eight DQA eplets (45V, 61G, 66I, 80S, 156F, 160AE, 175E and 175Q) and one DPA eplet (86T) that are monomorphic at another locus. These eplets should be considered self and therefore non-immunogenic because each recipient always has an allele on another locus with the same eplet.

\section{Discussion}

Although most class II eplets are on alleles controlled by a single HLA-DR, HLA-DQ or HLA-DP locus [39], this report describes our findings about interlocus class II eplets on alleles encoded by combinations of two or three loci. This concept is analogous to the sharing of interlocus class I eplets on combinations of HLA-A, -B and/or - $C$ alleles and their effect on donor-recipient compatibility. So far, interlocus class II eplets have received little attention but with the new abilities of clinical histocompatibility testing laboratories to do high-resolution typing of the entire HLA-DR, -DQ, -DP repertoire there are now opportunities to address this issue.

The inclusion of interlocus class II eplets is important for evaluating donor-recipient compatibility. For example, let us consider the well-documented, antibody-verified rp58E eplet (Table 1). This interlocus eplet is shared between DRB1*11 and multiple DPB alleles including DPB1*02, DPB1*03, DPB1*04:02 and more. Matching for eplets can be determined by two criteria: acceptability for the sensitized patient and permissibility for the non-sensitized patient. First, consider the sensitized patient who has a specific antibody induced by a rp58E-carrying allele such as DRB1*11 or DPB1*02:01; in such case the antibody producer's HLA type must have alleles without $\mathrm{rp58 \textrm {E }}$. If a recipient has developed rp58E-specific antibodies induced by DRB1*11:01 sensitization, then all rp58E-carrying DPB alleles should also be considered unacceptable mismatches although the recipient may have never been exposed to these DPB alleles. This interlocus DPB mismatch unacceptability can be determined only if the potential donor has also been DPB typed.

Conversely, rp58E-specific antibodies can also be induced by a perhaps unknown rp58E-carrying DPB allele; in such case, the reactive DR11 alleles have also become unexpectedly unacceptable mismatches although the recipient has never been exposed to them.

Eplet matching can also assess permissibility for non-sensitized recipients. In the rp58E example, any donor DRB or DPB allele with rp58E must be considered a non-permissible mismatch if all recipient's alleles lack that eplet. Conversely, all other donor alleles without rp58E can be considered permissible mismatches for that eplet.

A complete assessment of class II mismatch acceptability and permissibility should include both locus-specific and interlocus eplets, especially those that have been antibody-verified. The update of the International HLA Epitope Registry (http://www.epregistry.com.br) has now considerable 
numbers of antibody-verified eplets controlled by the separate HLA-DR, HLA-DQ and HLA-DP loci. The Registry has a new database for interlocus class II eplets that incorporates the information shown in Table 1, Table 2, Table 3, Table 4, Table 5, Table 6 and Table S4. The HLAMatchmaker website (www.epitopes.com) has been updated with a class II antibody analysis program that includes interlocus eplets.

The question can be raised why the information about antibody-verified interlocus class II eplets is so limited. One might respond that we never looked mainly because antibody producers and immunizers have rarely been typed completely at the DRB1/3/4/5, DQA/B, DPA/B loci at the 4digit allele level. Although the interlocus class II eplets listed in Table 6 represent theoretical speculations, their experimental verification with informative antibodies has now become feasible in clinical histocompatibility laboratories that require high-resolution DRDQDP typing of antibody producer and immunizer. An excellent source would be human monoclonal antibodies produced by cloned B-cells and tested with single allele panels. Allosera from sensitized patients can also be useful especially if the testing includes absorption-elution studies with informative interlocuseplet expressing alleles controlled by different loci. Furthermore, mutated alleles with residue substitutions will yield additional information.

Inclusion of interlocus structural comparisons will permit more accurate determinations of class II eplet-based matching important in clinical transplantation. Until now, compatibility has been assessed for separate loci but a better approach would be to consider the DR+DQ+DP repertoire as a single system analogous to matching for HLA-ABC at the eplet level.

\section{Additional Materials}

The following additional materials are uploaded at the page of this paper.

1. Table S1: Amino acid residues combinations with positive BLOSUM scores.

2. Table S2: Results of a BLAST analysis between DRB1*01:01, DQB1*02:01 and DPB1*01:01 sequences.

3. Table S3: Allele sharing between interlocus class II eplets and amino acid residues within a 3.5 Ångstrom radius.

4. Table S4: Results of a BLAST analysis between DRA1*01:01, DQA1*01:01 and DPA1*01:03 sequences.

5. Table S5: Amino acid residues within a 3.5 Ångstroms of $\beta$ chain eplets that are monomorphic at other loci.

6. Table S6: Amino acid residues within a 3.5 Ångstroms of alpha chain eplets that are monomorphic at other loci.

\section{Author Contributions}

RJD developed the interlocus concept and together with MM wrote the manuscript

\section{Competing Interests}

The authors have no conflicts of interest to disclose. 


\section{References}

1. Thaunat O, Hanf W, Dubois V, McGregor B, Perrat G, Chauvet C, et al. Chronic humoral rejection mediated by anti-HLA-DP alloantibodies: Insights into the role of epitope sharing in donor-specific and non-donor specific alloantibodies generation. Transpl Immunol. 2009; 20: 209-211.

2. Singh P, Colombe BW, Francos GC, Martinez Cantarin MP, Frank AM. Acute humoral rejection in a zero mismatch deceased donor renal transplant due to an antibody to an HLA-DP alpha. . Transplantation. 2010; 90: 220-221.

3. Wiebe C, Nickerson P. Acceptable mismatching at the class II epitope level: The Canadian experience. Curr Opin Organ Transplant. 2014; 19: 442-446.

4. Mierzejewska B, Schroder PM, Baum CE, Blair A, Smith C, Duquesnoy RJ, et al. Early acute antibody-mediated rejection of a negative flow crossmatch 3rd kidney transplant with exclusive disparity at HLA-DP. Hum Immunol. 2014; 75: 703-708.

5. Wiebe C, Nevins TE, Robiner WN, Thomas W, Matas AJ, Nickerson PW. The synergistic effect of class II HLA epitope-mismatch and nonadherence on acute rejection and graft survival. Am J Transplant. 2015; 15: 2197-2202.

6. Sapir-Pichhadze R, Tinckam K, Quach K, Logan AG, Laupacis A, John R, et al. HLA-DR and -DQ eplet mismatches and transplant glomerulopathy: A nested case-control study. Am J Transplant. 2015; 15: 137-148.

7. Filippone EJ, Farber JL. Humoral immunity in renal transplantation: Epitopes, CW and DP, and complement activating capability - an update. Clin Transplant. 2015; 29: 279-287.

8. Chin N, Westall G, Paraskeva M, Ciciulla J, Cantwell L, Snell G. Challenges inherent to the diagnosis of antibody-mediated rejection in lung transplantation. Respirol Case Rep. 2015; 3: 36-39.

9. Bryan CF, Chadha V, Warady BA. Donor selection in pediatric kidney transplantation using DR and DQ eplet mismatching: A new histocompatibility paradigm. Pediatr Transplant. 2016; 20: 926-930.

10. Walton DC, Hiho SJ, Cantwell LS, Diviney MB, Wright ST, Snell GI, et al. HLA matching at the eplet level protects against chronic lung allograft dysfunction. Am J Transplant. 2016; 16: 2695-2703.

11. Singh $\mathrm{P}$, Filippone EJ, Colombe BW, Shah AP, Zhan $T$, Harach $M$, et al. Sensitization trends after renal allograft failure: The role of $D Q$ eplet mismatches in becoming highly sensitized. Clin Transplant. 2016; 30: 71-80.

12. Pouliquen $E$, Baltzinger $P$, Lemle $A$, Chen CC, Parissiadis A, Borot $S$, et al. Anti-donor HLA antibody response after pancreatic islet grafting: Characteristics, risk factors, and impact on graft function. Am J Transplant. 2017; 17: 462-473.

13. Iwami D, Hotta $K$, Sasaki $H$, Hirose $T$, Higuchi $H$, Takada $Y$, et al. Highly immunogenic DQB1 mismatch eplets are associated with development of chronic active antibody-mediated rejection: A first report from Japan. Transplant Proc. 2017; 49: 84-87.

14. Wiebe C, Pochinco D, Blydt-Hansen TD, Ho J, Birk PE, Karpinski M, et al. Class II HLA epitope matching-a strategy to minimize de novo donor-specific antibody development and improve outcomes. Am J Transplant. 2013; 13: 3114-3122. 
15. Kishikawa $H$, Kinoshita $T$, Hashimoto $M$, Fukae $S$, Taniguchi $A$, Yamanaka $K$, et al. Class II HLA eplet mismatch is a risk factor for de novo donor-specific antibody development and antibody-mediated rejection in kidney transplantation recipients. Transplant Proc. 2018; 50: 2388-2391.

16. Wiebe C, Kosmoliaptsis V, Pochinco D, Gibson IW, Ho J, Birk PE, et al. A HLA-DR/DQ molecular mismatch: A prognostic biomarker for primary alloimmunity. Am J Transplant. 2018; 19: 17081719.

17. Wiebe C, Kosmoliaptsis V, Pochinco D, Taylor C, Nickerson P. A comparison of HLA molecular mismatch methods to determine HLA immunogenicity. Transplantation. 2018; 102, 1338-1343.

18. Walton DC, Cantwell L, Hiho S, Ta J, Wright S, Sullivan LC, et al. HLA class II eplet mismatch predicts de novo DSA formation post lung transplant. Transpl Immunol. 2018; 51: 73-75.

19. Sypek M, Kausman J, Holt S, Hughes P. HLA epitope matching in kidney transplantation: An overview for the general nephrologist. Am J Kidney Dis. 2018; 71: 720-731.

20. Willicombe M, Blow M, Santos-Nunez E, Freeman C, Brookes P, Taube D. Terasaki epitope mismatch burden predicts the development of de novo dq donor-specific antibodies and are associated with adverse allograft outcomes. Transplantation. 2018; 102: 127-134.

21. Ekong UD, Antala S, Bow L, Sese D, Morotti R, Rodriguez-Davalos M, et al. HLA, non-hla antibodies, and eplet mismatches in pediatric liver transplantation: Observations from a small, single-center cohort. Exp Clin Transplant. 2019; 17: 6-17.

22. Duquesnoy RJ, Askar M. HLAMatchmaker: A molecularly based algorithm for histocompatibility determination V. Eplet matching for HLA-DR, HLA-DQ and HLA-DP. Hum Immunol. 2007; 68: 12-25.

23. Altschul S, Madden T, Schäffer A, Zhang J, Zhang Z, Miller W, et al. Gapped BLAST and PSIBLAST: A new generation of protein database search programs. Nucleic Acids Res. 1997; 25: 3389-3402.

24. Henikoff S, Henikoff JG. Amino acid substitution matrices from protein blocks. Proc Natl Acad Sci USA. 1992; 89: 10915-10919.

25. Thorne JL, Goldman N, Jones DT. Combination of protein evolution and secondary structure. Mol Biol Evolution. 1996; 13: 666-673.

26. Hogue C. Cn3D: A new generation of three-dimensional molecular structure viewer. Trends Biochem Sci. 1997; 22: 314-316.

27. Bodmer J, Bodmer W, Heyes J, So A, Tonks S, Trowsdale J, et al. Identification of HLA-DP polymorphism with DP alpha and DP beta probes and monoclonal antibodies: Correlation with primed lymphocyte typing. Proc Natl Acad Sci USA. 1987; 84: 4596-4600.

28. Pistillo MP, Mazzoleni O, Kun L, Falco M, Tazzari PL, Ferrara GB. Production of two human hybridomas secreting antibodies to HLA-DRw11 and--DRw8+w12 specificities. Hum Immunol. 1991; 31: 86-93.

29. Yu WY, Watts R, Karr RW. Identification of amino acids in HLA-DPw4b and -DR5b1 chains that are involved in antibody binding epitopes using site-directed mutagenesis and DNA-mediated gene transfer. Hum Immunol. 1990; 27: 122-135.

30. Klohe E, Pistillo MP, Ferrara GB, Goeken NE, Greazel NS, Karr RW. Critical role of HLA-DR beta 1 residue 58 in multiple polymorphic epitopes recognized by xenogeneic and allogeneic antibodies. Hum Immunol. 1992; 35: 18-28. 
31. Diaz $G$, Amicosante $M$, Jaraquemada $D$, Butler $R H$, Gullen $M V$, Sanchez $M$, et al. Functional analysis of HLA-DP polymorphism: A crucial role for DPB residues 9, 11, 35, 55, 56, 69 and 8487 in T cell allorecognition and peptide binding. Int Immunol. 2003; 15: 565-576.

32. Vaidya S, Hilson B, Sheldon S, Cano P, Fernandez-Vina M. DP reactive antibody in a zero mismatch renal transplant pair. Hum Immunol. 2007; 68: 947-949.

33. Marshall WH, Drover S, Codner D, Gamberg J, Copp MD, Liu HW, et al. HLA-DP epitope typing using monoclonal antibodies. Hum Immunol. 1998; 59: 189-197.

34. Cano P, Fernandez-Vina M. Two sequence dimorphisms of DPB1 define the immunodominant serologic epitopes of HLA-DP. Hum Immunol. 2009; 70: 836-843.

35. Tongio MM, Van der Berg Loonen E, Bignon JD, Chandanayingyong D, Dormoy A, Eiermann T, et al. HLA-DP detected by serology. In: Genetic diversity of HLA functional and medical implication: Proceedings of the twelfth international histocompatibility workshop and conference. 2ed. Paris, France: EDK; 1997. p. 135-138.

36. Inoko H, Bodmer JG, Heyes JM, Drover S, Trowsdale J, Marshall WH. Joint report on the transfectant/monoclonal antibody component. In: HLA 1991 proceedings of the eleventh international histocompatibility workshop and conference. Oxford, UK: Oxford University Press; 1991. p. 919-930.

37. Drover S, Gamberg J, Qi C, Marshall WH. Identification of a shared determinant on DQB1*0201 and DRB1*09, DRB1*1001 and DRB1*1401 monoclonal antibody. Hum Immunol. 1992; 34: 11.

38. Callender CJ, Fernandez-Vina M, Leffell MS, Zachary AA. Frequency of HLA-DP-specific antibodies and a possible new cross-reacting group. Hum Immunol. 2012; 73: 175-179.

39. Duquesnoy RJ, Marrari M, da Mata Sousa LCD, Marroquim M, do Monte SJH. Brief report: second update the HLA-DR,-DQ,-DP eplet database of the International registry of HLA epitopes. Hum Immunol. 2019; Submitted.

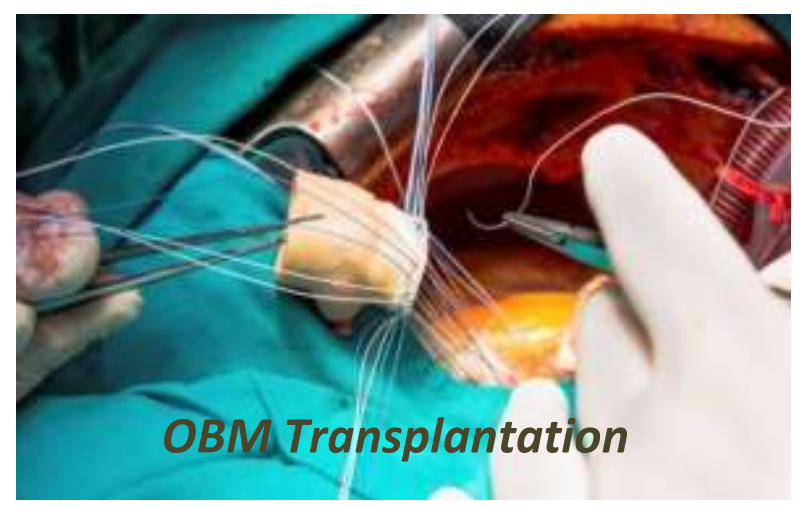

Enjoy OBM Transplantation by:

1. Submitting a manuscript

2. Joining in volunteer reviewer bank

3. Joining Editorial Board

4. Guest editing a special issue

For more details, please visit:

http://www.lidsen.com/journals/transplantation 